\title{
Impact of Early Childhood Caries and Maternal Behaviors on Oral Health-Related Quality of Life of Children
}

\author{
Joanna Tatith Pereira ${ }^{1}$, Jessica Klöckner Knorst² ${ }^{\circledR}$, Patrícia Blaya Luz ${ }^{\circledR}$, Inaiá Bonfadini ${ }^{1}$, \\ Monique Scapinello ${ }^{3}$, Fernando Neves Hugo ${ }^{4}$, Fernando Borba de Araujo ${ }^{5(0)}$
}

\begin{abstract}
${ }^{1}$ Faculty of Dentistry, Federal University of Rio Grande do Sul, Porto Alegre, RS, Brazil.
${ }^{2}$ Department of Stomatology, School of Dentistry, Federal University of Santa Maria, Santa Maria, RS, Brazil.

${ }^{3}$ Institute of Psychology, Federal University of Rio Grande do Sul, Porto Alegre, RS, Brazil.

${ }^{4}$ Public Health Dentistry Department, Federal University of Rio Grande do Sul, Porto Alegre, RS, Brazil.

${ }_{5}^{5}$ Pediatric Dentistry Department, Federal University of Rio Grande do Sul, Porto Alegre, RS, Brazil.
\end{abstract}

Author to whom correspondence should be addressed: Joanna Tatith Pereira, Rua Joana Dar'c, 488, Nossa Senhora de Lourdes, Santa Maria, RS, Brazil. 97060-360. Phone: + 5551981714591 . E-mail: contato@joanna.odo.br.

Academic Editors: Alessandro Leite Cavalcanti and Wilton Wilney Nascimento Padilha

Received: 01 October 2019 / Accepted: 25 March 2020 / Published: 13 April 2020

How to cite this article: Pereira JT, Knorst JK, Luz PB, Bonfadini I, Scapinello M, Hugo FN, et al. Impact of early childhood caries and maternal behaviors on oral health-related quality of life of children. Pesqui Bras Odontopediatria Clín Integr. 2020; 20:e5283. https://doi.org/10.1590/pboci.2020.065

\begin{abstract}
Objective: To evaluate the impact of early childhood caries, maternal behaviors and associated factors on the oral health-related quality of life (OHRQoL) of preschool children. Material and Methods: This crosssectional study was conducted with 163 preschool children aged 3-4 old from Porto Alegre, Southern Brazil. Mothers completed the Brazilian version of the Early Childhood Oral Health Impact Scale (ECOHIS) and answered questions about sociodemographic and maternal behaviors characteristics. The severity of dental caries was assessed by ICDAS criteria for evaluated early childhood caries (ECC). A Poisson regression model was used to investigate the association of ECC and associated factors on OHRQoL. Results: A total of $91.4 \%$ of preschooler children was ECC (ICDAS $\geq 1$ ). Mean ECOHIS function domain and parental anxiety domain scores were significantly higher in children with dental caries $(\mathrm{p}<0.05)$. The adjusted Poisson regression model showed that household income, maternal education, mother's job status and breastfeeding time was significantly associated with ECOHIS scores. Regarding oral conditions, child caries experience was associated with worsening child and family quality of life (RR 2.21; 95\% IC: 1.43-3.41). Conclusion: Our findings suggest that ECC, socioeconomic and maternal behaviors characteristics influence children's OHRQoL.
\end{abstract}

Keywords: Dental Caries; Maternal Behavior; Quality of Life; Observational Study. 


\section{Introduction}

In the last years has been observed a change in the pattern of distribution and occurrence of childhood caries [1-5]. However, its prevalence is still high, reaching $55 \%$ in the deciduous dentition and $60 \%$ in the permanent dentition [6]. In Brazil, the most recent epidemiological survey reports that 53.4\% of 5-year-old children have experience of dental caries [7]. Therefore, early childhood caries (ECC) in preschool children is an important dental public health problem in Brazil.

Dental caries has been consistently associated with a negative impact on the oral function, social and psychological wellbeing of the children and their families, influencing the oral health-related quality of life (OHRQoL) [8-13]. The concept of OHRQoL refers to the impact that oral conditions have on individual's dayto-day functioning, well-being and overall quality of life [14,15]. OHRQoL measures have emerged as the main health outcome, both for clinical trials and for observational studies, allowing health professionals to evaluate the effectiveness of their treatment and the quality of care provided from the patients' perspective [14$17]$.

Interest in assessing OHRQoL in preschool children has grown. Low socioeconomic status and maternal education are directly related with poor oral health status and worse OHRQoL [9,10,18-21]. Similarly, maternal behaviors are associated with poor oral health in preschool children [22-24]. To our knowledge, few studies have evaluated the association of ECC and maternal health behaviors in a critical period of childhood development with the perception of mothers in relation to the OHRQoL of their children. Moreover, most studies evaluated the presence of caries using DMFT, which does not discriminate cavitated lesions from the initial stages of dental caries [10-25]. In addition, approach to these issues in children and families enrolled in primary healthcare services was not explored yet.

Although health-related behaviors are important in determining health status, a focus on behaviors alone will not address social gradients in population oral health. Furthermore, impacts caused by dental caries and their consequences do not reflect only in childhood, but may persist throughout life [26]. The understanding that knowledge, health beliefs and attitudes, patterns of behavior and psychosocial factors are interlinked and influence in determining individuals' responses, the aim of this study was to evaluate the impact of ECC, maternal behaviors and associated factors on OHRQoL of preschool children and their parents. We hypothesized that children who presented ECC are more likely to report low OHRQoL.

\section{Material and Methods}

Study Design and Sample

This cross-sectional study included 163 preschools born in 2008 in Porto Alegre, a southern city in Brazil. Whose families were registered in one of 12 Public Primary Care Services, located in Porto Alegre (RS, Brazil). All children born in 2008 and their mothers were initially eligible for the study if they resided in public health care services (PHC) catchment areas. Sampling occurred randomly by clusters, maintaining the proportion of live births in each of the 12 PHC. For this, each PHC provided the list of 2008 live births in order of date of birth and, from a table of random numbers, the children and their mothers were selected and invited to participate.

The sample size was calculated with consideration of the following parameters: sampling error of $5 \%$, $95 \%$ confidence interval $(95 \% \mathrm{CI})$, the number of live births in these communities in 2008 (674 infants) and a prevalence of $31 \%$ in the exposed group (with caries - ECC at two years) [27]. The ratio of exposed to unexposed was 1:1. Considering possible losses, 20\% was added to the sample size, resulting in a required 
minimum of 208 subjects. Children and/or mothers who had systemic diseases that prevent them from participating in the study or cognitive disabilities, and mothers who were not speakers of the Brazilian Portuguese Language were excluded.

\section{Data Collection}

Data collection was conducted from December 2011 to January 2013, including clinical examination of the children, and structured interviews conducted with the mothers. Four previously trained and calibrated examiners conducted the examinations in the children's home. Telephone contact was made with the person legally responsible for the child to invite the respective mothers to participate in the study. An instruction manual for field research was prepared and used during training and data collection. A pilot study of 30 pairs of children and mothers (who were not part of the sample) was carried out to test the methodology and the understanding of questionnaires used.

The Brazilian version of the Early Childhood Oral Health Impact Scale (ECOHIS) was used to assess the children OHRQoL - the outcome of this study [28-30]. Mothers answered the questionnaire through a face-to-face interview conducted by previously trained interviewers. The ECOHIS was used to measure the impact of oral health on the children's and his/her family's quality of life. The ECOHIS consists of 13 items, 9 including a child impact section and 4 a family impact section. Answers were recorded using a Likert scale with response options coded 0-5. The mean ECOHIS scores were calculated by overall scores ranging from 0 to 36 . Higher overall scores indicating poorer OHRQoL. Questionnaires with more than 2 "do not know" responses were excluded from the sample.

Children's sociodemographic characteristics were collected from mothers through a self-administered questionnaire regarding sex, maternal educational level, household income, household crowding, marital status and mother's job status. Maternal education was collected in years of study and categorized as $<9$ years (incomplete primary education), 9-11 years, or $>11$ years. Household income was a sum of all forms of income in a month. It was collected in Reais (Brazilian cure - $\mathrm{R} \$ 3.75$ it was equivalent to $\mathrm{U} \$ 1.00$ approximately) and categorized as the Brazilian minimum wages $(\mathrm{BMW}): \leq 2,>2 \leq 5$, or $>5$ times the BMW. The amount of individuals in the house was evaluated through the number of people living in the house and categorized according to the median $(\leq 4$ people, or $>4$ ). The marital status was dichotomized in not married or married. The mother's job status was evaluated thought the working time outside home $(>30$ hours per week or $<30$ hours per week) in the first two years of the child's life.

Questions related to maternal behavior included: "Did you breastfeed your child? If so, for how long?" (Collected in months and categorized as $\leq 2$ years or $>2$ years, according to WHO recommendations) [30]; "Do you agree that your child is able to brush his or her teeth alone?" (Dichotomized and yes or no); "How often do you perform oral brushing of your child's teeth?" and the following possible responses: $0=$ more than once daily; $1=$ once daily and $2=$ occasionally or never.

For the evaluation of clinical conditions, children were examined in their homes using sterile dental mirrors and probes, an air compressor and portable lighting. First, dental prophylaxis with a toothbrush and floss was performed in each child. Personal protective equipment was used to ensure biosafety. The severity of dental caries experience was assessed by ICDAS criteria of dried and illuminated dental surfaces. These were categorized according to the worst score: caries-free (ICDAS 0), enamel lesions (ICDAS 1-2-3), and dentin lesions (ICDAS 4-5-6). Data were also dichotomized as the presence of cavity related to caries: caries-free and 
non-cavitated lesions (ICDAS 0-1-2), and cavitated lesions (ICDAS > 3) [31,32]. ECC was deemed present if a child had at least one surface with an ICDAS score of 1 or more.

In the evaluation of Traumatic dental injury (TDI), the criterion used was the Children's Dental Health Survey Criteria - CDHS-UK [33]. Data were dichotomized according to the presence of at least one type of trauma or absence. Malocclusion was recorded as anterior open bite, increased overjet, deep bite, anterior crossbite and/or posterior crossbite [34]. The evaluation was carried out with the child in occlusion, and malocclusion was deemed to be present if at least one of the conditions was present.

\section{Statistical Analysis}

Statistical analysis was performed using SPSS V.17.0 (SPSS Inc., Chicago, IL, USA). Mean ECOHIS scores were described for each domain in relation to the exposures of interest. Kruskal-Wallis and MannWhitney tests with a significance level of $5 \%(\mathrm{p}<0.05)$ were used. Poisson regression with robust variance was performed using ECOHIS scores as outcome and socioeconomic characteristics, maternal behaviors, and oral conditions as exposures. All variables that had $\mathrm{p}<0.20$ in the crude analyses (and those with clinical or epidemiological importance) were included in the final model. From this comparison, a fully adjusted model was obtained. Rate ratios (RR) and respective $95 \%$ confidence intervals (CI) for the exposures in relation to the outcomes are shown.

\section{Ethical Issues}

This study was approved by the Ethics Committee for Research of the Conceição Hospital Group (CEP-GHC/11-196). Before entering the study, all mothers read and signed an informed consent form.

\section{Results}

A total of 208 mother-infant pairs were invited to participate; $163(78.4 \%)$ agreed to participate. The reasons for losses included the inability to find the children/mother $(n=34)$ and refusal to take part in the study $(\mathrm{n}=11)$. Inter and intraexaminer agreement (Kappa values) for dental caries ranged from 0.64 to 0.73 and from 0.75 to 0.80 , respectively. For trauma and malocclusion, examiners achieved a Kappa equal to 1.0.

Table 1 summarizes the characteristics of the sample according to sociodemographic, behavioral and oral health variables. Most children are girls (55.6\%) and were in the lower-income categories. For maternal behaviors, $18.4 \%$ of mothers reported having breastfed their children after 2 years of age. Regarding dental caries, the most (91.4\%) preschooler children was ECC (the presence of at least one clinically detected carious lesion (ICDAS $\geq 1)$. The ICDAS ranged between 0 and $6(2.54 \pm 1.57)$. Dental trauma and malocclusions were present in $38 \%$ and $46 \%$ of the children, respectively.

Table 1. Characteristics of participants according to sociodemographic, behavioral and oral health variables.

\begin{tabular}{lccc}
\hline & Variables & N & \% \\
\hline Sex & & & \\
$\quad$ Male & 74 & 45.4 \\
$\quad$ Female & 89 & 55.6 \\
Maternal Education & & \\
$\quad$ < 9 Years & 40 & 24.5 \\
9-11 Years & 72 & 44.2 \\
$>11$ Years & 51 & 31.3
\end{tabular}




\begin{tabular}{|c|c|c|}
\hline \multicolumn{3}{|l|}{ Household Income } \\
\hline$\leq 2 \mathrm{BMW}^{\mathrm{a}}$ & 56 & 34.4 \\
\hline$>2 \leq 5 \mathrm{BMW}$ & 79 & 48.4 \\
\hline$>5 \mathrm{BMW}$ & 28 & 17.2 \\
\hline \multicolumn{3}{|l|}{ Number of Individuals in the House } \\
\hline$\leq 4$ People & 82 & 50.3 \\
\hline$>4$ People & 81 & 49.7 \\
\hline \multicolumn{3}{|l|}{ Mother's Working Time Outside Home } \\
\hline$>30$ Hours & 97 & 59.5 \\
\hline$\leq 30$ Hours & 66 & 40.5 \\
\hline \multicolumn{3}{|l|}{ Marital Status } \\
\hline Not Married & 59 & 36.2 \\
\hline Married/Living Together & 104 & 63.8 \\
\hline \multicolumn{3}{|l|}{ Breastfeeding Time } \\
\hline$\leq 2$ Years & 133 & 81.6 \\
\hline$>2$ Years & 30 & 18.4 \\
\hline \multicolumn{3}{|c|}{ Agree that the Child is Able to Brush his or her Teeth Alone } \\
\hline Yes & 27 & 16.6 \\
\hline No & 136 & 83.4 \\
\hline \multicolumn{3}{|l|}{ Frequency of Child' Oral Brushing } \\
\hline More Than Once Daily & 82 & 50.3 \\
\hline Once Daily & 60 & 36.8 \\
\hline Occasionally or Never & 21 & 12.9 \\
\hline \multicolumn{3}{|l|}{ Early Childhood Caries } \\
\hline Caries-free (ICDAS o) & 14 & 8.6 \\
\hline Enamel lesions (ICDAS 1, 2 or 3 ) & 111 & 68.1 \\
\hline Dentin lesions (ICDAS 4, 5 or 6 ) & 38 & 23.3 \\
\hline Cavitated lesions (ICDAS > 3) & 52 & 32.0 \\
\hline \multicolumn{3}{|l|}{ Traumatic Dental Injury } \\
\hline Presence & 62 & 38.0 \\
\hline Absence & 101 & 62.0 \\
\hline \multicolumn{3}{|l|}{ Malocclusion } \\
\hline With & 75 & 46.0 \\
\hline Without & 88 & 54.0 \\
\hline
\end{tabular}

Table 2 shows the descriptive distribution of total ECOHIS and domain scores. About 48.0\% of mothers reported that their children had an impact on at least one of the items of the ECOHIS. The negative impacts on OHRQoL were more prevalent in the child subscale $(74 / 163 ; 45.4 \%)$ than in the family subscale (39/163; 23.9\%). Oral pain and toothache were the more prevalent impacts reported in the child subscale, while guilt was the most prevalent impact in the family subscale.

The mean ECOHIS overall and domains scores according to socioeconomic, behaviors and clinical characteristics of the sample are presented in Table 3. The ECOHIS scores ranged between 0 and 28, with an average of $2.15(\mathrm{SD}=3.85)$. The highest scores were 16 and 12 on the child and family subscales, respectively. When the overall mean of ECOHIS was analyzed, ECC was associated with a negative impact on OHRQoL $(\mathrm{p}<0.05)$. There were significant differences in mean ECOHIS self-image and social interaction domain scores (child subscale) in relation to maternal schooling, family income, prolonged breastfeeding and tooth brushing frequency $(\mathrm{p}<0.05)$. Mean ECOHIS function domain (child subscale) and parental anxiety domain (family subscale) scores also differed significantly in relation to the presence of cavitated lesions and severity of dental caries $(\mathrm{p}<0.05)($ Table 3$)$. 
Table 2. Descriptive distribution of total ECOHIS and domains scores.

\begin{tabular}{|c|c|c|c|c|c|c|c|}
\hline \multirow[b]{2}{*}{ ECOHIS Domains } & \multicolumn{6}{|c|}{ Responses } & \multirow[b]{2}{*}{ Mean (SD) } \\
\hline & $\begin{array}{l}\text { Never } \\
\mathrm{N}(\%)\end{array}$ & $\begin{array}{l}\text { Hardly Ever } \\
\qquad \mathrm{N}(\%)\end{array}$ & $\begin{array}{l}\text { Occasionally } \\
\mathrm{N}(\%)\end{array}$ & $\begin{array}{l}\text { Often } \\
\mathrm{N}(\%)\end{array}$ & $\begin{array}{l}\text { Very Often } \\
\quad \mathrm{N}(\%)\end{array}$ & $\begin{array}{l}\text { Don't Know } \\
\quad \mathrm{N}(\%)\end{array}$ & \\
\hline \multicolumn{8}{|l|}{ Child Section } \\
\hline 1. Has your child had pain in the teeth, mouth or jaws? (Symptom) & $103(63.2)$ & $36(22.1)$ & $20(12.3)$ & $3(1.8)$ & $1(0.6)$ & $0(0.0)$ & $0.55(0.8)$ \\
\hline $\begin{array}{l}\text { 2. Has your child had difficulty drinking hot or cold beverages because } \\
\text { of dental problems or dental treatments? (Function) }\end{array}$ & $148(90.8)$ & $10(6.1)$ & $3(1.8)$ & $0(0.0)$ & $1(0.6)$ & $1(0.6)$ & $0.15(0.6)$ \\
\hline $\begin{array}{l}\text { 3. Has your child had difficulty eating some foods because of dental } \\
\text { problems or dental treatments? (Function) }\end{array}$ & $147(90.2)$ & $13(8.0)$ & $1(0.6)$ & $3(1.8)$ & $0(0.0)$ & $0(0.0)$ & $0.13(0.4)$ \\
\hline $\begin{array}{l}\text { 4. Has your child had difficulty pronouncing any words because of } \\
\text { dental problems or dental treatments? (Function) }\end{array}$ & $152(93.3)$ & $3(1.8)$ & $13(1.8)$ & $2(1.2)$ & $3(1.8)$ & $0(0.0)$ & $0.17(0.7)$ \\
\hline $\begin{array}{l}\text { 5. Has your child missed preschool, daycare or school because of dental } \\
\text { problems or dental treatments? (Function) }\end{array}$ & $149(91.4)$ & $8(4.9)$ & $4(2.5)$ & $0(0.0)$ & $0(0.0)$ & $2(1.2)$ & $0.16(0.7)$ \\
\hline $\begin{array}{l}\text { 6. Has your child had trouble sleeping because of dental problems or } \\
\text { dental treatments? (Psychology) }\end{array}$ & $155(95.1)$ & $4(2.5)$ & $2(1.2)$ & $1(0.6)$ & $1(0.6)$ & $\mathrm{o}(0.0)$ & $0.09(0.5)$ \\
\hline $\begin{array}{l}\text { 7. Has your child been irritable or frustrated because of dental } \\
\text { problems or dental treatments? (Psychology) }\end{array}$ & $14.1(86.5)$ & $15(9.2)$ & $5(3.1)$ & $2(1.2)$ & $0(0.0)$ & $0(0.0)$ & $0.19(0.5)$ \\
\hline $\begin{array}{l}\text { 8. Has your child avoided smiling or laughing because of dental } \\
\text { problems or dental treatments? (Self-image/social interaction) }\end{array}$ & $157(96.3)$ & $4(2.5)$ & $2(1.2)$ & $0(0.0)$ & $\mathrm{o}(0.0)$ & $\mathrm{o}(0.0)$ & $0.05(0.3)$ \\
\hline $\begin{array}{l}\text { 9. Has your child avoided talking because of dental problems or dental } \\
\text { treatments? (Self-image/social interaction) }\end{array}$ & $157(96.3)$ & $2(1.2)$ & $3(1.8)$ & $1(0.6)$ & $\mathrm{o}(0.0)$ & $\mathrm{o}(0.0)$ & $0.07(0.4)$ \\
\hline \multicolumn{8}{|l|}{ Family Section } \\
\hline $\begin{array}{l}\text { 10. Have you or another family member been upset because of your } \\
\text { child's dental problems or dental treatment? (Parental distress) }\end{array}$ & $147(90.2)$ & $11(6.7)$ & $3(1.8)$ & $0(0.0)$ & $2(1.2)$ & $\mathrm{o}(0.0)$ & $0.15(0.6)$ \\
\hline $\begin{array}{l}\text { 11. Have you or another family member felt guilty because of your } \\
\text { child's dental problems or dental treatment? (Parental distress) }\end{array}$ & $139(85.3)$ & $9(5.5)$ & $8(4.9)$ & $2(1.2)$ & $5(3.1)$ & $\mathrm{o}(0.0)$ & $0.31(0.9)$ \\
\hline $\begin{array}{l}\text { 12. Have you or another family member taken time off from work } \\
\text { because of your child's dental problems or dental treatment? (Family } \\
\text { function) }\end{array}$ & $155(95.1)$ & $4(2.5)$ & $2(1.2)$ & $1(0.6)$ & $1(0.6)$ & $0(0.0)$ & $0.09(0.5)$ \\
\hline $\begin{array}{l}\text { 13. Has your child had dental problems or dental treatments that had a } \\
\text { financial impact on your family? (Family function) }\end{array}$ & $158(96.9)$ & $3(1.8)$ & $2(1.2)$ & $\mathrm{o}(0.0)$ & $0(0.0)$ & $0(0.0)$ & $0.04(0.3)$ \\
\hline
\end{tabular}


Table 3. Mean ECOHIS overall and domains scores according to socioeconomic, behaviors and clinical characteristics.

\begin{tabular}{|c|c|c|c|c|c|c|c|}
\hline \multirow{3}{*}{ Variables } & \multicolumn{6}{|c|}{ Domains } & \multirow{3}{*}{$\begin{array}{c}\text { ECOHIS } \\
\text { Mean (SD) }\end{array}$} \\
\hline & SD & FD & PD & SSD & PDD & FFD & \\
\hline & Mean (SD) & Mean (SD) & Mean (SD) & Mean (SD) & Mean (SD) & Mean (SD) & \\
\hline \multicolumn{8}{|l|}{ Socioeconomic Variables } \\
\hline \multicolumn{8}{|l|}{ Maternal Education } \\
\hline$<9$ Years & $0.67(0.88)$ & $0.27(0.51)$ & $0.27(0.66)$ & $0.22(0.51)$ & $0.42(0.88)$ & $0.06(0.32)$ & $0.28(0.47)$ \\
\hline 9-11 Years & $0.52(0.85)$ & $0.12(0.26)$ & $0.08(0.22)$ & - & $0.15(0.47)$ & $0.11(0.37)$ & $0.13(0.19)$ \\
\hline$>11$ Years & $0.47(0.73)$ & $0.09(0.21)$ & $0.11(0.35)$ & $0.00(0.07)$ & $0.18(0.61)$ & $0.00(0.07)$ & $0.11(0.19)$ \\
\hline p-value & 0.541 & 0.310 & 0.369 & $0.008^{+}$ & 0.117 & 0.118 & 0.396 \\
\hline \multicolumn{8}{|l|}{ Household Income } \\
\hline$\leq 2 \mathrm{MW}$ & $0.69(0.85)$ & $0.25(0.45)$ & $0.22(0.58)$ & $0.13(0.42)$ & $0.34(0.78)$ & $0.13(0.45)$ & $0.25(0.41)$ \\
\hline$>2 \leq 5 \mathrm{MW}$ & $0.48(0.82)$ & $0.11(0.26)$ & $0.10(0.30)$ & $0.02(0.15)$ & $0.22(0.62)$ & $0.04(0.19)$ & $0.13(0.21)$ \\
\hline$>5 \mathrm{MW}$ & $0.42(0.74)$ & $0.07(0.16)$ & $0.08(0.23)$ & - & $0.03(0.13)$ & - & $0.07(0.09)$ \\
\hline p-value & 0.149 & 0.063 & 0.582 & $0.046^{\dagger}$ & 0.051 & 0.125 & 0.067 \\
\hline \multicolumn{8}{|c|}{ Number of Individuals in the House } \\
\hline$\leq 4$ People & $0.58(0.88)$ & $0.13(0.29)$ & $0.14(0.37)$ & $0.04(0.27)$ & $0.21(0.61)$ & $0.10(0.40)$ & $0.16(0.30)$ \\
\hline$>4$ People & $0.50(0.76)$ & $0.16(0.38)$ & $0.14(0.45)$ & $0.07(0.27)$ & $0.25(0.67)$ & $0.02(0.13)$ & $0.16(0.28)$ \\
\hline p-value & & 0.828 & 0.702 & 0.157 & 0.651 & 0.117 & 0.555 \\
\hline \multicolumn{8}{|c|}{ Mother's Working Time Outside Home } \\
\hline$>30$ Hours & $0.54(0.81)$ & $0.19(0.38)$ & $0.15(0.48)$ & $0.06(0.30)$ & $0.25(0.71)$ & $0.04(0.24)$ & $0.18(0.33)$ \\
\hline$<30$ Hours & $0.54(0.84)$ & $0.09(0.23)$ & $0.12(0.30)$ & $0.05(0.21)$ & $0.19(0.51)$ & $0.09(0.37)$ & $0.14(0.22)$ \\
\hline p-value & 0.980 & 0.119 & 0.859 & 0.616 & 0.700 & 0.314 & 0.830 \\
\hline \multicolumn{8}{|l|}{ Marital Status } \\
\hline Not Married & $0.67(0.91)$ & $0.16(0.33)$ & $0.18(0.55)$ & $0.05(0.32)$ & $0.26(0.75)$ & $0.11(0.43)$ & $0.20(0.37)$ \\
\hline Married/Living Together & $0.47(0.76)$ & $0.14(0.34)$ & $0.11(0.31)$ & $0.05(0.24)$ & $0.21(0.57)$ & $0.03(0.18)$ & $0.14(0.24)$ \\
\hline p-value & 0.173 & 0.454 & 0.974 & 0.533 & 0.869 & 0.187 & 0.386 \\
\hline \multicolumn{8}{|l|}{ Behaviors Variables } \\
\hline \multicolumn{8}{|l|}{ Breastfeeding } \\
\hline$\leq 2$ Years & $0.51(0.78)$ & $0.14(0.33)$ & $0.12(0.40)$ & $0.03(0.21)$ & $0.19(0.57)$ & $0.03(0.16)$ & $0.14(0.24)$ \\
\hline$>2$ Years & $0.70(0.98)$ & $0.17(0.36)$ & $0.20(0.48)$ & $0.15(0.43)$ & $0.41(0.88)$ & $0.21(0.59)$ & $0.25(0.45)$ \\
\hline p-value & 0.294 & 0.918 & 0.457 & $0.020^{\dagger}$ & $0.038^{+}$ & $0.015^{+}$ & 0.324 \\
\hline
\end{tabular}


Agree that the child is able to brush his or

her teeth alone

\begin{tabular}{|c|c|c|c|c|c|c|c|}
\hline Yes & $0.59(0.79)$ & $0.19(0.47)$ & $0.25(0.68)$ & $0.05(0.28)$ & $0.18(0.60)$ & $0.03(0.13)$ & $0.18(0.34)$ \\
\hline No & $0.53(0.83)$ & $0.14(0.30)$ & $0.11(0.34)$ & $0.05(0.27)$ & $0.24(0.65)$ & $0.07(0.32)$ & $0.16(0.28)$ \\
\hline p-value & 0.534 & 0.788 & 0.316 & 0.770 & 0.497 & 0.930 & 0.706 \\
\hline \multicolumn{8}{|c|}{ requency of Child' Oral Brushing } \\
\hline More than Once a Day & $0.52(0.86)$ & $0.10(0.23)$ & $0.07(0.25)$ & $0.01(0.11)^{\mathrm{A}}$ & $0.09(0.27)$ & $0.06(0.23)$ & $0.11(0.16)$ \\
\hline Once Daily & $0.53(0.76)$ & $0.22(0.44)$ & $0.20(0.47)$ & $0.08(0.34)^{\mathrm{AB}}$ & $0.36(0.85)$ & $0.09(0.41)$ & $0.22(0.38)$ \\
\hline Occasionally or Never & $0.66(0.85)$ & $0.13(0.29)$ & $0.21(0.68)$ & $0.16(0.42)^{\mathrm{B}}$ & $0.38(0.86)$ & & $0.20(0.37)$ \\
\hline p-value & 0.682 & 0.297 & 0.144 & $0.035^{*}$ & 0.073 & 0.702 & 0.545 \\
\hline
\end{tabular}

Oral Conditions

Early Childhood Caries

aries

Enamel Lesions

Dentin Lesions

$0.12(0.21)^{\mathrm{A}}$

$0.14(0.30)$

$0.00(0.00)$

$0.00(0.00)^{\mathrm{A}}$

$0.10(0.28)$

$0.12(0.16)^{\mathrm{A}}$

$0.13(0.35)^{\mathrm{A}}$

$0.08(0.25)$

$0.03(0.18)$

$0.13(0.39)^{\mathrm{B}}$

$0.02(0.14)$

$0.11(0.19)^{\mathrm{A}}$

$0.78(0.93)$

$0.037^{*}$

$.30(0.71)$

$0.14(0.46)$

$0.59(1.07)^{\mathrm{C}}$

$0.17(0.53)$

$0.31(0.47)^{\mathrm{B}}$

Caries-free/Non-cavitated Lesions

$0.45(0.77)$

$0.11(0.27)$

$0.07(0.21)$

0.148

0.001 *

0.062

0.027 *

Cavitated Lesions

$0.73(0.90)$

$0.23(0.42)$

0.27 (0.65)

$0.02(0.16)$

$0.13(0.39)$

$0.04(0.18)$

$0.11(0.17)$

0.052

$0.025^{\dagger}$

0.062

0.054

$0.45(0.95)$

$0.12(0.46)$

$0.27(0.43)$

Traumatic Dental Injury

Presence

$0.46(0.76)$

$0.16(0.36)$

$0.18(0.49)$

$0.07(0.31)$

$0.003+$

0.302

$0.015^{\dagger}$

Absence

$0.67(0.90)$

0.13 (0.28)

$0.06(0.21)$

$0.03(0.17)$

$0.23(0.64)$

$0.05(0.33)$

$0.17(0.33)$

0.095

0.789

0.427

0.22 (0.64)

0.08 (0.24)

$0.15(0.21)$

Malocclusion

\begin{tabular}{|c|c|c|c|c|c|c|c|}
\hline With & $0.61(0.90)$ & $0.11(0.26)$ & $0.15(0.39)$ & $0.06(0.30)$ & $0.22(0.62)$ & $0.08(0.36)$ & $0.16(0.31)$ \\
\hline Without & $0.46(0.72)$ & $0.19(0.40)$ & $0.12(0.44)$ & $0.04(0.23)$ & $0.24(0.66)$ & $0.04(0.20)$ & $0.16(0.27)$ \\
\hline $\mathrm{p}$-value & 0.367 & 0.312 & 0.395 & 0.622 & 0.857 & 0.515 & 0.578 \\
\hline
\end{tabular}

SD: Symptoms Domain; FD: Function Domain; PD: Psychology Domain; SSD: Self-Image/Social Interaction Domain; PDD: Parental Distress Domain; FFD: Family Function Domain; ECOHIS: Overall; *KruskalWallis test; ${ }^{+}$Mann-Whitney test; The different letters mean statistically significant difference between groups. 
Table 4 shows the results of adjusted Poisson regression analyses. In relation some socioeconomic variables, high maternal education (RR 0.38; 95\% IC: 0.21-0.69) were associated with better OHRQoL. In addition, children whose mothers worked in their first two years of life (RR 0.54; 95\% IC: 0.30-0.96) presented the highest impacts on OHRQoL. In relation the behaviors characteristics, prolonged breastfeeding (RR 2.15; 95\% CI: 1.16-3.96) was associated with higher scores on the ECOHIS. Regarding oral conditions, dental caries was associated with the outcome (RR 2.21; 95\% IC: 1.43-3.41), where children with ECC presented highs ECOHIS scores, and consequently, poorest OHRQoL.

Table 4. Rate ratio (RR) for unadjusted and adjusted association between overall ECOHIS scores and explored variables.

\begin{tabular}{|c|c|c|c|c|}
\hline \multirow[t]{2}{*}{ Variables } & \multicolumn{2}{|c|}{ Unadjusted } & \multicolumn{2}{|c|}{ Adjusted } \\
\hline & $\operatorname{RR}(95 \% \mathrm{CI})$ & p-value & $\mathrm{RR}(95 \% \mathrm{CI})$ & p-value \\
\hline \multicolumn{5}{|l|}{ Demographic Variables } \\
\hline Sex & & 0.57 & \# & \\
\hline Female & 1.00 & & & \\
\hline Male & $1.16(0.67-2.01)$ & & & \\
\hline \multicolumn{5}{|l|}{ Socioeconomic Variables } \\
\hline Maternal Education & & 0.173 & & $<0.01$ \\
\hline$<9$ Years & 1.00 & & 1.00 & \\
\hline 9-11 Years & $0.53(0.28-1.02)$ & & $0.49(0.30-0.81)$ & \\
\hline$>11$ Years & $0.54(0.23-1.30)$ & & $0.38(0.21-0.69)$ & \\
\hline Household Income ${ }^{c}$ & & $<0.01$ & & \\
\hline$\leq 2$ Minimum Wages & 1.00 & & $\#$ & \\
\hline$>2 \leq 5$ Minimum Wages & $0.61(0.37-0.99)$ & & & \\
\hline$>5$ Minimum Wages & $0.36(0.18-0.75)$ & & & \\
\hline Number of Individuals in the House & & 0.78 & & \\
\hline$\leq 4$ People & 1.00 & & $\#$ & \\
\hline$>4$ People & $0.92(0.50-1.67)$ & & & \\
\hline Mother's Working time Outside Homed & & 0.15 & & $<0.05$ \\
\hline$>30$ Hours & 1.00 & & 1.00 & \\
\hline$<30$ Hours & $0.66(0.38-1.16)$ & & $0.54(0.30-0.96)$ & \\
\hline Marital Status & & 0.97 & & \\
\hline Not Married & 1.00 & & & \\
\hline Married/Living Together & $0.99(0.59-1.65)$ & & \# & \\
\hline \multicolumn{5}{|l|}{ Behaviors Variables } \\
\hline \multicolumn{5}{|l|}{ Breastfeeding } \\
\hline$\leq 2$ Years & 1.00 & $<0.05$ & 1.00 & $<0.05$ \\
\hline$>2$ Years & $2.03(1.12-3.69)$ & & $2.15(1.16-3.96)$ & \\
\hline $\begin{array}{l}\text { Agree that the child is able to brush his } \\
\text { or her teeth alone }\end{array}$ & & 0.54 & & \\
\hline Yes & 1.00 & & $\#$ & \\
\hline No & $0.82(0.44-1.53)$ & & & \\
\hline Frequency of Child' Oral Brushing & & $<0.05$ & & 0.06 \\
\hline More Than Once a Day & 1.00 & & 1.00 & \\
\hline Once Daily & $1.66(1.03-2.65)$ & & $1.54(0.98-2.42)$ & \\
\hline Occasionally or Never & $1.58(0.76-3.25)$ & & $1.40(0.69-2.86)$ & \\
\hline \multicolumn{5}{|l|}{ Clinical Conditions } \\
\hline Early Childhood Caries & & $<0.01$ & & $<0.01$ \\
\hline Caries-free/Non-cavitated Lesions & 1.00 & & 1.00 & \\
\hline Cavitated Lesions & $2.18(1.34-3.54)$ & & $2.2(1.43-3.41)$ & \\
\hline Traumatic Dental Injury & & 0.60 & & \\
\hline Presence & $1.13(0.70-1.80)$ & & \# & \\
\hline
\end{tabular}


Absence

Malocclusion

With

Without

RR: Rate Ratio; CI: Confidence Interval.
1.00

$1.48(0.91-2.40)$

1.00

\section{Discussion}

The present findings support the hypothesis that ECC influences the children's OHRQoL. Children who presented ECC in the form of cavitated lesions was significantly associated with the mothers' negative perception in relation to their children's OHRQoL. Similarly, lower maternal education, mother's job status and prolonged breastfeeding were associated with more negative impacts on OHRQoL. Understanding these associations may lead to a broader view of the aspects that influence children's and their families' quality of life.

The presence of ECC influenced negatively children's OHRQoL, in accordance with previous studies $[10,13,17,35-37]$, thus providing strong evidence that disease severity is directly proportional to the negative impact generated in OHRQoL. The negative influences of cavitated dental caries on children's quality of life include changes in behavior, chewing and sleeping difficulties, decrease in school performance and mainly dental pain [8,10,38]. Pain impacts daily activities and affects the physical status of the children, as well as their psychological well-being and social interactions, negatively influence the OHRQoL [5,39,40]. Moreover, cavitated lesions can leave parents feeling guilty and upset about the oral condition of their children, which reflects in the OHRQoL $[10,41]$.

Children with white spot lesions presented the lowest scores on ECOHIS and, consequently, a better OHRQoL. This result is in agreement with previous studies that showed that initial lesions of caries do not negatively impact OHRQoL [36,37], including overtime [37]. An explanation for this is that initial dental caries does not usually cause pain, discomfort or aesthetic concerns in the children and their parents, it is understandable that children with only these types of lesions do not report impacts on OHRQoL [37]. However, although the presence of non-cavitated lesions was not associated with the evaluated outcomes, studies have shown that the history of the disease is a risk factor for dental caries [42].

Our findings demonstrated that low maternal education impacted negatively the children OHRQoL, which corroborates with similar findings in the literature [13,14]. A possible explanation for this is that families and children with better socioeconomic conditions generally have better behaviors related to oral health, greater access to health care and preventive interventions, and consequently may have a better quality of life $[13,41]$. Therefore, it is important to reconsider the current paradigm related to OHRQoL restricted to oral conditions, including contemporary concepts of disease and its consequences, emphasizing the importance of relating these to patients' individual, social, and environmental OHRQoL [13,42].

The mother's job status was also associated with children's OHRQoL. Preschoolers whose mothers worked in their first two years of life, presented the highest impacts on OHRQoL. Studies report that children whose mothers are housewives have lower rates of caries compared to those mothers who work away from home [43]. This may be because mothers who have regular jobs have less time to perform routine oral hygiene and care of their children than mothers who stay at home [44]. Taken together, these results add to the existing evidence about the relation between behaviors and OHRQoL.

In relation to breastfeeding, children who have been breastfed for more than 2 years presented higher scores on the ECOHIS. Breastfeeding up to 2 years of age or longer is uncommon in Western countries (on average, a prevalence of 33\%) [30] mainly due to lack of policies that encourage prolonged breastfeeding, 
which coincides with the findings of this study $(18.4 \%$ of the women breastfed their children more than 2 years). The affective link between mother and child is established in the first 24 months of the child's life, and it is a result of constant interactions held between the pair since birth [44]. When the interaction succeeds, the mother is able to understand the demands of the child, which aids physical and mental development and enables better knowledge of the mother about her child [44].

The findings of this study should be interpreted with accounting for some limitations. The crosssectional design precludes the assessment of risk factors of impacts on OHRQoL. In addition, dental caries was evaluated through the ICDAS index, which requires a longer and more detailed training, also to involving critical steps during the examination. However, the findings obtained through the ICDAS, which mainly include the presence of non-cavitated lesions, show more realistic ECC distribution. In addition, the ICDAS has been previously used in other epidemiological surveys with children [11,38,45] and showed the ease of use and processing of data gathered, when compared with the widely used DMFT [32].

\section{Conclusion}

Caries severity, family's socioeconomic characteristics and maternal behaviors were associated with an impact on the OHRQoL of preschool children. The results may help in improving our understanding about how oral conditions affect preschool-aged children's quality of life. The prevention and control of early childhood caries through public policies and on the part of pediatric dentists are fundamental to the prevention of negative impacts on the quality of life of children.

\section{Authors' Contributions}

\begin{tabular}{|c|c|c|}
\hline JTP & (iD) $0000-0002-2434-4305$ & $\begin{array}{l}\text { Conceptualization, Methodology, Investigation, Writing - Original Draft } \\
\text { Preparation and Writing - Review and Editing. }\end{array}$ \\
\hline JKK & (iD) 0000-0001-7792-8032 & Writing - Original Draft Preparation and Writing - Review and Editing. \\
\hline PBL & (iD) $0000-0001-8204-4231$ & $\begin{array}{l}\text { Conceptualization, Methodology, Investigation, Writing - Original Draft } \\
\text { Preparation and Writing - Review and Editing. }\end{array}$ \\
\hline IB & (iD) $0000-0001-9466-0488$ & Investigation and Validation and Writing - Review and Editing. \\
\hline MS & (iD) 0000-0003-2701-0092 & Investigation and Validation and Writing - Review and Editing. \\
\hline $\mathrm{FNH}$ & (iD) 0000-0003-2222-7719 & Formal Analysis, Validation and Writing - Review and Editing. \\
\hline FBA & (iD) 0000-0003-2892-3256 & Conceptualization, Methodology and Writing - Review and Editing. \\
\hline
\end{tabular}

\section{Financial Support}

Universal Public Notice - CNPQ Notice 479894/2010-8, Researcher Gaucho Public Notice - FAPERGS 111368-4 and by the Higher Education Personnel Improvement Coordination (CAPES).

\section{Conflict of Interest}

The authors declare no conflicts of interest.

\section{References}

[1] Gimenez T, Bispo BA, Souza DP, Viganó ME, Wanderley MT, Mendes FM, et al. Does the decline in caries prevalence of Latin American and Caribbean children continue in the new century? Evidence from systematic review with meta-analysis. PLoS One 2016; 11(10):e0164903. https://doi.org/10.1371/journal.pone.0164903

[2] Frencken JE, Sharma P, Stenhouse L, Green D, Laverty D, Dietrich T. Global epidemiology of dental caries and severe periodontitis - a comprehensive review. J Clin Periodontol 2017; 44(Suppl 18):S94-S105. https://doi.org/10.1111/jcpe.12677 
[3] Pitts NB, Ramon RJ, Baez J, Diaz-Guillory C, Donly KJ, et al. Early Childhood Caries: IAPD Bangkok Declaration. J Dent Child 2019; 86(2):72.

[4] Tinanoff N, Baez RJ, Guillory CD, Donly JK, Feldens CA, McGrath C, et al. Early childhood caries epidemiology, aetiology, risk assessment, societal burden, management, education, and policy: global perspective. Int J Paediatr Dent 2019; 29(3):238-48. https://doi.org/10.1111/ipd.12484

[5] Lotto M, Strieder AP, Ayala Aguirre PE, Andrade Moreira Machado MA, Rios D, et al. Parental perspectives on early childhood caries: a qualitative study. Int J Paediatr Dent 2020; https://doi.org/10.1111/ipd.12622

[6] Kassebaum NJ, Bernabé E, Dahiya M, Bhandari B, Murray CJ, Marcenes W. Global burden of untreated caries: a systematic review and metaregression. J Dent Res 2015; 94(5):650-8. https://doi.org/10.1177/0022034515573272

[7] Brasil. Ministério da Saúde. Pesquisa Nacional de Saúde Bucal. SB Brasil 2010. Resultados Principais. Brasília: Ministério da Saúde; 2011; 92pp. [In Portuguese].

[8] Pahel BT, Rozier RG, Slade GD. Parental perceptions of children's oral health: the Early Childhood Oral Health Impact Scale (ECOHIS). Health Qual Life Outcomes 2007; 5:6. https://doi.org/10.1186/1477-7525-5-6

[9] Oliveira LB, Sheiham A, Bonecker M. Exploring the association of dental caries with social factors and nutritional status in Brazilian preschool children. Eur J Oral Sci 2008; 116(1):37-43. https://doi.org/10.1111/j.1600-0722.2007.00507.x.

[10] Abanto J, Carvalho TS, Mendes FM, Wanderley MT, Bonecker M, Raggio DP. Impact of oral diseases and disorders on oral health-related quality of life of preschool children. Community Dent Oral Epidemiol 2011; 39(2):105-14. https://doi.org/10.1111/j.1600-0528.2010.00580.x

[11] de Amorim RG, Figueiredo MJ, Leal SC, Mulder J, Frencken JE. Caries experience in a child population in a deprived area of Brazil, using ICDAS II. Clin Oral Investig 2012; 16(2):513-20. https://doi.org/10.1007/s00784-011-0528-9

[12] Guedes RS, Piovesan C, Antunes JL, Mendes FM, Ardenghi TM. Assessing individual and neighborhood social factors in child oral health-related qualityof life: a multilevel analysis. Qual Life Res 2014; 23(9):2521-30. https://doi.org/10.1007/s11136-014-0690-Z

[13] Chaffee BW, Rodrigues PH, Kramer PF, Vítolo MR, Feldens CA. Oral health-related quality-of-life scores differ by socioeconomic status and caries experience. Community Dent Oral Epidemiol 2017; 45(3):216-24. https://doi.org/10.1111/cdoe.12279

[14] Locker D, Aleen F. What do measures of 'oral health-related quality of life' measure? Community Dent Oral Epidemiol 2007; 35(6):401-11. https://doi.org/10.1111/j.1600-0528.2007.00418.x

[15] Sischo L, Broder HL. Oral health-related quality of life: what, why, how, and future implications. J Dent Res 2011; 90(11):1264-70. https://doi.org/10.1177/0022034511399918

[16] McGrath C, Broder H, Wilson-Genderson M. Assessing the impact of oral health on the life quality of children: implications for research and practice. Community Dent Oral Epidemiol 2004; 32(2):81-5. https://doi.org/10.1111/j.1600-0528.2004.00149.x

[17] Kramer PF, Feldens CA, Ferreira SH, Bervian J, Rodrigues PH, Peres MA. Exploring the impact of oral diseases and disorders on quality of life of preschool children. Community Dent Oral Epidemiol 2013; 41(4):327-35. https://doi.org/10.1111/cdoe.12035

[18] Goettems ML, Ardenghi TM, Romano AR, Demarco FF, Torriani DD. Influence of maternal dental anxiety on oral health-related quality of life of preschool children. Qual Life Res 2011; 20(6):951-9.

https://doi.org/10.1007/s11136-010-9816-0

[19] Scarpelli AC, Oliveira BH, Tesch FC, Leao AT, Pordeus IA, Paiva SM. Psychometric properties of the Brazilian version of the Early Childhood Oral Health Impact Scale (B-ECOHIS). BMC Oral Health 2011; 11:19. https://doi.org/10.1186/1472-6831-11-19

[20] Correa-Faria P, Paixao-Goncalves S, Paiva SM, Martins-Junior PA, Vieira-Andrade RG, Marques LS, et al. Dental caries, but not malocclusion or developmental defects, negatively impacts preschoolers' quality of life. Int J Paediatr Dent 2016; 26(3):211-9. https://doi.org/10.1111/ipd.12190

[21] Knorst JK, Menegazzo GR, Emmanuelli B, Mendes FM, Ardenghi TM. Effect of neighborhood and individual social capital in early childhood on oral health-related quality of life: a 7-year cohort study. Qual Life Res 2019; 28(7):177382. https://doi.org/10.1007/s11136-019-02138-4

[22] Skeie MS, Espelid I, Riordan PJ, Klock KS. Caries increment in children aged 3-5 years in relation to parents' dental attitudes: Oslo, Norway 2002 to 2004. Community Dent Oral Epidemiol 2008; 36(5):441-50. https://doi.org/10.1111/j.1600-0528.2008.00430.x

[23] Begzati A, Bytyci A, Meqa K, Latifi-Xhemajli B, Berisha M. Mothers' behaviours and knowledge related to caries experience of their children. Oral Health Prev Dent 2014; 12(2):133-40. https://doi.org/10.3290/j.ohpd.a3 1667

[24] Yang AJ, Gromoske AN, Olson MA, Chaffin JG. Single and cumulative relations of social risk factors with children's dental health and care - utilization within regions of the United States. Matern Child Health J 2016; 20(3):495-506. https://doi.org/10.1007/s10995-015-1847-2

[25] de Paula JS, Sarracini KL, Meneghim MC, Pereira AC, Ortega EM, Martins NS, et al. Longitudinal evaluation of the impact of dental caries treatment on oral health-related quality of life among schoolchildren. Eur J Oral Sci 2015; 123(3):173-8. https://doi.org/10.1111/eos.12188 
[26] Holst D, Schuller AA. Oral health in a life-course: birth-cohorts from 1929 to 2006 in Norway. Community Dent Health 2012; 29(2):134-43.

[27] Ferreira SH, Beria JU, Kramer PF, Feldens EG, Feldens CA. Dental caries in 0- to 5-year-old Brazilian children: prevalence, severity, and associated factors. Int J Paediatr Dent 2007; 17(4):289-96.

https://doi.org/10.1111/j.1365-263X.2007.00831.x

[28] Martins-Junior PA, Ramos-Jorge J, Paiva SM, Marques LS, Ramos-Jorge ML. Validations of the Brazilian version of the Early Childhood Oral Health Impact Scale (ECOHIS). Cad Saúde Pública 2012; 28(2):367-74. https://doi.org/10.1590/So102-311X2012000200015

[29] Tesch FC, Oliveira BH, Leao A. Semantic equivalence of the Brazilian version of the Early Childhood Oral Health Impact Scale. Cad Saúde Pública 2008; 24(8):1897-1909. https://doi.org/10.1590/So 102-311X2008000800018

[30] Delgado C, Matijasevich A. Breastfeeding up to two years of age or beyond and its influence on child growth and development: a systematic review. Cad Saúde Pública 2013; 29(2):243-56.

https://doi.org/10.1590/So102-311X2013000200012

[31] Pitts N. "ICDAS"--an international system for caries detection and assessment being developed to facilitate caries epidemiology, research and appropriate clinical management. Community Dent Health 2004; 21(3):193-8.

[32] Mendes FM, Braga MM, Oliveira LB, Antunes JL, Ardenghi TM, Bonecker M. Discriminant validity of the International Caries Detection and Assessment System (ICDAS) and comparability with World Health Organization criteria in a cross-sectional study. Community Dent Oral Epidemiol 2010; 38(5):398-407. https://doi.org/10.1111/j.1600-0528.2010.00557.x

[33] Traebert J, Marcon KB, Lacerda JT. Prevalence of traumatic dental injuries and associated factors in schoolchildren of Palhoca, Santa Catarina State. Cien Saúde Coletiva 2010; 15(Suppl 1):1849-55. https://doi.org/10.1590/s1413-81232010000700098

[34] Grabowski R, Stahl F, Gaebel M, Kundt G. Relationship between occlusal findings and orofacial myofunctional status in primary and mixed dentition. Part I: Prevalence of malocclusions. J Orofac Orthop 2007; 68(1):26-37. https://doi.org/10.1007/s00056-007-1606-0

[35] Scarpelli AC, Paiva SM, Viegas CM, Carvalho AC, Ferreira FM, Pordeus IA. Oral health-related quality of life among Brazilian preschool children. Community Dent Oral Epidemiol 2013; 41(4):336-44. https://doi.org/10.1111/cdoe.12022

[36] Ramos-Jorge J, Pordeus IA, Ramos-Jorge ML, Marques LS, Paiva SM. Impact of untreated dental caries on quality of life of preschool children: different stages and activity. Community Dent Oral Epidemiol 2014; 42(4):311-22. https://doi.org/10.1111/cdoe.12086

[37] Guedes RS, Ardenghi TM, Piovesan C, Emmanuelli B, Mendes FM. Influence of initial caries lesions on quality of life in preschool children: a 2-year cohort study. Community Dent Oral Epidemiol 2016; 44(3):292-300. https://doi.org/10.1111/cdoe.12217

[38] Sheiham A. Dental caries affects body weight, growth and quality of life in pre-school children. Br Dent J 2006; 201(10):625-6. https://doi.org/10.1038/sj.bdj.4814259

[39] Ortiz FR, Tomazoni F, Oliveira MD, Piovesan C, Mendes F, Ardenghi, TM. Toothache, associated factors, and its impact on Oral Health-Related Quality of Life (OHRQoL) in preschool children. Braz Dent J 2014; 25(6):546-53. https://doi.org/10.1590/0103-6440201302439

[40] Pesaressi E, Villena RS, Frencken JE. Dental caries and oral health-related quality of life of 3-year-olds living in Lima, Peru. Int J Paediatr Dent 2020; 30(1):57-65. https://doi.org/10.1111/ipd.12582

[41] Gomes MC, Pinto-Sarmento TC, Costa EM, Martins CC, Granville-Garcia AF, Paiva SM. Impact of oral health conditions on the quality of life of preschool children and their families: a cross-sectional study. Health Qual Life Out 2014; 12:55. https://doi.org/10.1186/1477-7525-12-55

[42] Tinanoff N, Reisine S. Update on early childhood caries since the Surgeon General's Report. Acad Pediat 2009; 9(6):396-403. https://doi.org/10.1016/j.acap.2009.08.006

[43] de Paula JS, Leite IC, de Almeida AB, Ambrosano GM, Mialhe FL. The impact of socioenvironmental characteristics on domains of oral health-related quality of life in Brazilian schoolchildren. BMC Oral Health 2013; 13:10. https://doi.org/10.1186/1472-6831-13-10

[44] Castilho AR, Mialhe FL, Barbosa Tde S, Puppin-Rontani RM. Influence of family environment on children's oral health: a systematic review. J Pediatr 2013; 89(2):116-23. https://doi.org/10.1016/j.jped.2013.03.014.

[45] Guedes RS, Piovesan C, Ardenghi TM, Emmanuelli B, Braga MM, Mendes FM. Presence of initial caries lesions as a risk factor for caries in preschool children: a cohort study. Caries Res 2018; 52(1-2):32-41. https://doi.org/10.1159/000479824 